\title{
Transmission Electron Forward Scattered Diffraction and Low Voltage SEM/STEM Characterization of Binder-Free $\mathrm{TiO}_{2}$ Electrodes
}

\author{
Micah Sussman ${ }^{1}$, Nicolas Brodusch ${ }^{1}$, Raynald Gauvin ${ }^{1}$, and George P. Demopoulos ${ }^{1}$ \\ 1. Department of Mining and Materials Engineering, McGill University, Montreal, Quebec, Canada.
}

Graphite, the current commercial anode material in batteries suffers from the formation of a surface/electrolyte interface (SEI) layer that adversely affects performance. However, titania $\left(\mathrm{TiO}_{2}\right)$ does not form an SEI layer and is both abundant and inexpensive, making it a prime anode material replacement [1]. While much work has been done to determine the effects of morphology and phase of $\mathrm{TiO}_{2}$ on lithium intercalation, the effect of the method fabrication of the electrode on its electrochemical performance is still unknown. Currently, electrodes are fabricated using active material, conductive additives, and a non-electroactive polymer binder mixed mechanically with a solvent. In this work, we introduced a chemo-mechanical fabrication method that produces binder-free electrodes. Without binder, more loading of active material is possible. Further, agglomeration of both $\mathrm{TiO}_{2}$ and conductive additives that occurs with the binder-based method is limited by using dispersants. Finally, the electrode is sintered, forming a 3-dimensional mesoporous structure that provided a $25 \%$ increase in capacity over the binder-based method, as well as better capacity retention over multiple cycles.

Commercial P25 titania was used as the active material for this work. By applying transmission electron forward scattered diffraction (t-EFSD) [2] in a cold-field emission scanning electron microscope (CFE-SEM) with an accelerating voltage of $30 \mathrm{kV}$ to the P25 titania powder, the morphology and crystallographic structure of the various particles were assessed (Figure 1). This information coupled with particle size showed that the rutile was too large to lithiate successfully [3], thus lithium would insert only into anatase. After sintering, the binder-free electrode films were compared to the binder electrode films using low voltage secondary (SE) and backscattered electrons (BSE) imaging. At low voltages $\left(\mathrm{V}_{0}<2 \mathrm{kV}\right)$, the collected signals are generated from the shallow surface of the nano-objects under investigation and allows reducing charging and beam damage at the same time. Hence, they provide an accurate description of the objects shapes coupled with the chemical information carried by the BSEs. The low voltage images in Figure 2 show that the binder-free films undergo necking that provides better interparticle connections which allow the electrons flowing within the material during charge and discharge to reach a larger percentage of the material, allowing for more lithium to enter.

Further, connections to the conductive additive are increased due to the increased dispersion. Using the transmission mode of the CFE-SEM (STEM), the dispersion of conductive additives within the electrodes was quantified. Figure 3 shows representative $70-80 \mathrm{~nm}$ thick slices of the electrodes film observed at $30 \mathrm{kV}$ in bright-field STEM. Using image analysis, it was determined that the binder-free method had smaller, more numerous agglomerates $\left(75 \%\right.$ were $\left.<0.005 \mu \mathrm{m}^{2}\right)$, as compared to the standard method whose agglomerates were much larger $\left(60 \%\right.$ were $\left.<0.005 \mu^{2}\right)$. This, as well as the increased interparticle connectivity explains the $25 \%$ increased capacity and capacity retention.

\section{References:}

[1] T. Brezesinski et al, Journal of the American Chemical Society 129 (2009), pp. 1802-1809.

[2] N. Brodusch, H. Demers, and R. Gauvin, Journal of Microscopy 250 (2013), pp. 1-14.

[3] M. A. Reddy et al, Electrochemistry Communications 8 (2006), pp. 1299-1303. 

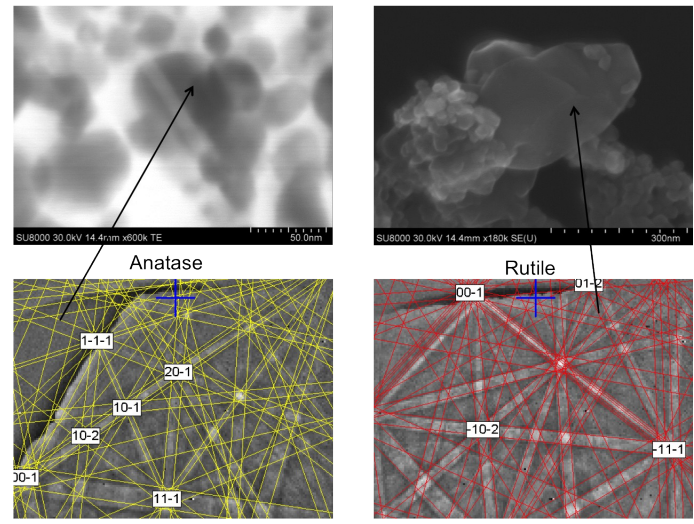

Figure 1. Top: Scanning transmission electron microscopy (STEM) bright field (top left) and secondary electron (top right) images of the P25 titania powder. Bottom: Transmission electron forward scattered diffraction patterns of two $\mathrm{TiO}_{2}$ particles from the P25 powder indexed as anatase (bottom left) and rutile (bottom right) crystals. The accelerating voltage was $30 \mathrm{kV}$.
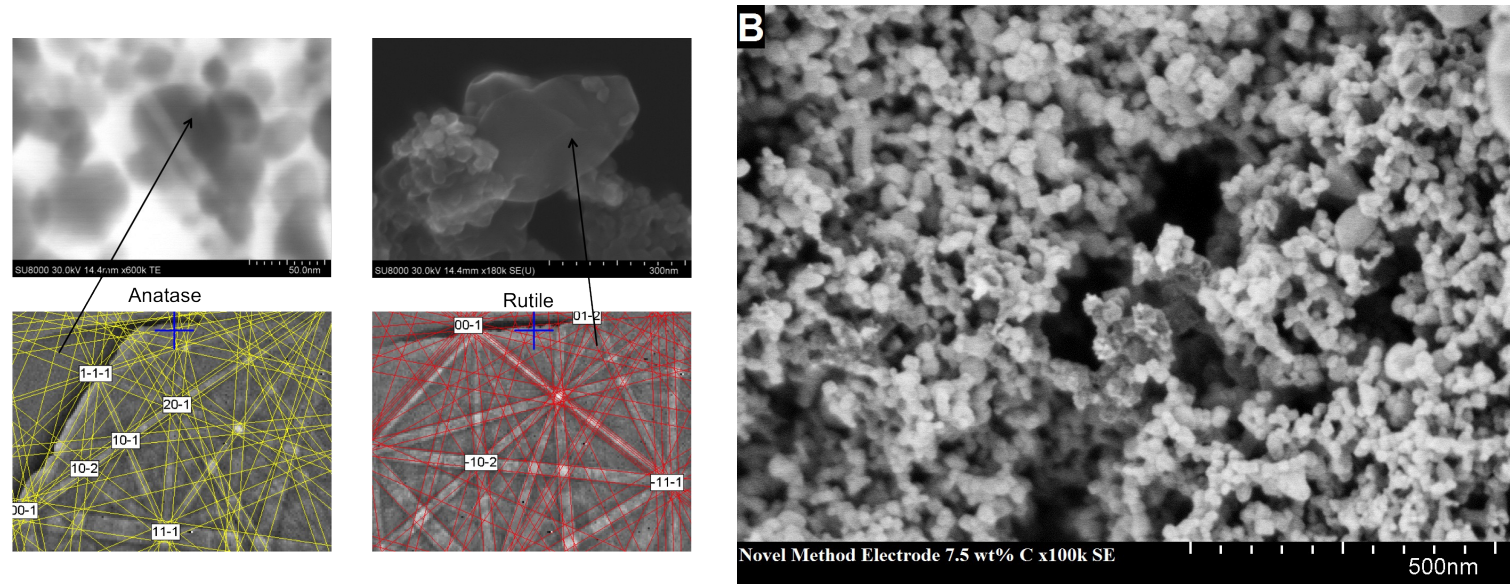

Figure 2. Low voltage secondary electron images of binder-based (A) and binder-free (B) electrode films showing the effects of sintering on particle interconnections.

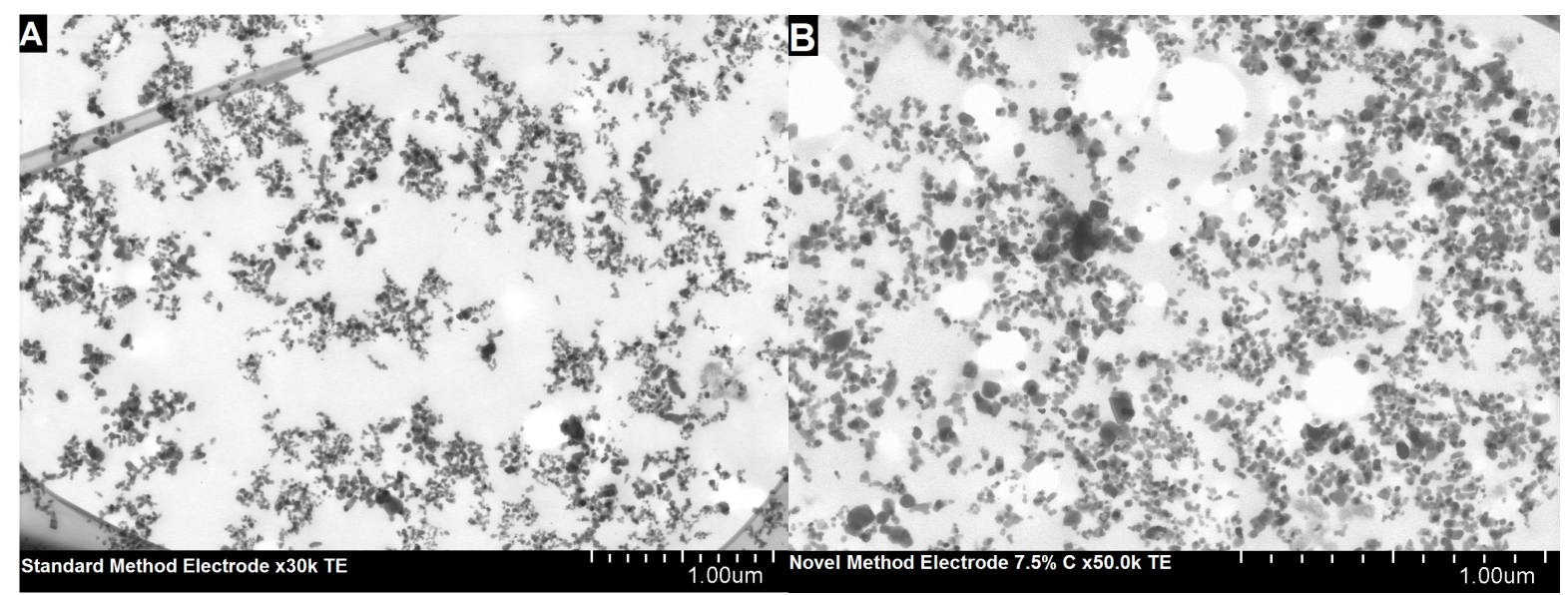

Figure 3. Representative bright-field STEM images at $30 \mathrm{kV}$ of 70-80 nm thick slices of binder-based (A) and binder-free (B) electrode films mounted in LR White resin and ultramicrotomed. 